\title{
Acoustic Characteristics of Voice in Patients with Chronic Kidney Disease
}

\author{
Fatma Mohammed Abd El-gaber' \\ Yossra Sallam iD ${ }^{2}$ \\ Hanaa Mohammed Eid El Sayed (iD) ${ }^{3}$ \\ 'Otorhinolaryngology Department, \\ Faculty of Medicine for Girls, Al-Zahraa \\ Hospital, Al Azhar University, Cairo, \\ Egypt; ${ }^{2}$ Phoniatrics, Otorhinolaryngology \\ Department, Faculty of Medicine for \\ Girls, Al-Zahraa Hospital, Al Azhar \\ University, Cairo, Egypt; ${ }^{3}$ Internal \\ Medicine Department, Faculty of \\ Medicine for Girls, Al-Zahraa Hospital, Al \\ Azhar University, Cairo, Egypt
}

Purpose: To investigate the multifactorial effects of chronic kidney disease (CKD) and hemodialysis (HD) on subjects' voices by examining correlations between laboratory investigations, respiratory function, and acoustic voice parameters.

Methods: This case-control study was conducted on 60 participants aged 18-50 years, divided equally into three groups: controls (no health problems or voice disorders), cCKD (stage 3-5, no HD HD]), HD, and CKD stage 5. The study took 21 months. All participants underwent general and otolaryngological examinations, followed by laboratory investigations (hemoglobin, uric acid, $\mathrm{HCO}_{3}$, estimated glomerular filtration rate, urea, urea-reduction ratio, and creatinine), respiratory function tests, and acoustic voice analysis.

Results: There were significant differences between the control and HD groups for jitter, shimmer, and harmonic:noise (HNR) ratio $(P=0$ and between the control and CKD groups for shimmer and HNR $(P=0)$, with no significant difference between HD and CKD. There were statistically significant correlations between duration of HD and HNR, jitter percentage, and shimmer percentage $(P=0$.

Conclusion: Systemic effects of CKD and HD were found to impair the acoustic characteristics of voice in both groups. Regression analysis revealed that hemoglobin, uric acid, and expiratory time were the most significant predictors of impaired acoustic characteristics.

Keywords: CKD, hemodialysis, voice changes, pulmonary functions, eGFR

\section{Introduction}

Normal voice production requires interactions among the power source, vibrator, and resonators. The power source (adequate respiratory support) compresses air and forces it toward the larynx. The vocal folds close and open, permitting small bursts of air to escape through them to produce mucosal vibrations. ${ }^{1}$ This complex dynamic process requires harmony between the respiratory and laryngeal muscles.

The lungs and kidneys are connected physiologically and pathologically in healthy individuals and patients with CKD, whether on hemodialysis (HD) or not. $^{2}$ Chronic kidney disease (CKD) is a beyond-repair condition that impedes the function of various body systems, particularly the respiratory system. The potency of the respiratory and laryngeal muscles, among generalized muscle weakness, is extensively impaired. ${ }^{3}$

CKD may also be complicated by pulmonary edema, pleurisy with subsequent chest pain and fibrothorax, predisposition to such infections as tuberculosis, pulmonary calcifications, and/or urinothorax. ${ }^{4}$ In addition, patients on HD often suffer generalized muscle weakness and fatigue that may affect muscles of respiration and phonation, which in turn will affect respiratory support and strongly impair voice quality. ${ }^{5}$ With an
Correspondence: Fatma Mohammed Abc El-gaber

Otorhinolaryngology Department Faculty of Medicine for Girls, Al-Zahraa Hospital, Al Azhar University, 12 lbrahimNagy Street, Nasr, Cairo I I824, Egypt Tel +20 I I-2445-4762

Email fatmamohamed30@hotmail.com 
estimated prevalence of $15.2 \%$ among patients on HD, the etiology of dysphonia among these patients should be completely unveiled. ${ }^{6}$

\section{Objectives}

This study aimed to investigate the multifactorial effects of CKD and HD on subjects' voices by examining correlations between laboratory investigations, respiratory function, and acoustic voice parameters.

\section{Methods}

This was a case-control study conducted on 60 participants presenting to the otorhinolaryngology and internal medicine outpatient clinics at Al-Zahraa University Hospital from September 2018 to June 2020.

participants were aged 18-50 years, and they were divided equally into three groups:

1. Control group: free from any systemic disorder or voice disorders.

2. CKD group: CKD stage 3-5 not on HD. They were diagnosed by nephrologists with clinical and laboratory investigations and estimated glomerular filtration rate (eGFR) using the Modification of Drugs in Renal Disease equation: ${ }^{7}$

eGFR $\left(\mathrm{mL} / \mathrm{min}\right.$ per $\left.1.73 \mathrm{~m}^{2}\right)=175 \times$ serum creatinine $-1.154 \times$ age $-0.203 \times 1.212$ (if patient is black) $\times 0.742$ (if female)

3. HD group (CKD stage 5): patients had been on HD $>1$ year. They underwent three sessions of HD per week, and every session took 4 hours.

All cases underwent full history-taking, general examination(weight and height, and head and neck examination, including, nose, pharynx, and larynx)laryngeal examination using rigid a $70^{\circ}$ endoscope (Karl Storz), acoustic analysis of the voice including (fundamental frequency $\left[f_{0}\right]$, shimmer percentage $[S \%]$, jitter percentage $[J \%]$, and harmonic:noise ratio $[\mathrm{HNR}]$ ) using a PreSonus Audio Electronics voice analyzerpulmonary function tests (vital capacity [VC], forced VC[FVC] forced expiratory volume in 1 second $\left[\mathrm{FEV}_{1}\right]$ forced expiratory flow $\left[\mathrm{FEF}_{25 \%} \mathrm{FEF}_{75 \%}\right]$, expiratory time, and $\mathrm{FEV}_{1} / \mathrm{FVC}$ )and laboratory investigations (hemoglobin, uric acid, $\mathrm{HCO}_{3} \mathrm{eGFR}$, urea, urea-reduction ratio and creatinine). Cases with a history of functional or organic voice disorders (eg, vocal abuse, nodules, or polyps), systemic diseases (bronchial asthma), smokers, females on hormonal therapy, and those who refused to participate were all excluded.

\section{Statistical Analysis}

Data were collected, revised, coded and entered into SPSS $20 .{ }^{8}$ Qualitative data are presented as numbers and percentages and quantitative data as means $\pm \mathrm{SD}$ and ranges when their distribution was parametric. Comparisons between two groups with qualitative data were done using \#x1D712; ${ }^{2}$ tests. Comparisons between two independent groups with quantitative data and parametric distribution was done using independent $t$-tests. ANOVA was used to analyze differences between means of the three studied groups. Post hoc tests were run to confirm differences between groups on oneway ANOVA showed statistically significant differences in group means. Pearson's correlation coefficient was used to measure correlations between quantitative variables. Linear regression analysis was done for prediction of acoustic parameters. The CI was set to $95 \%$ and the margin of error accepted set to $5 \%$. $P<0.05$ was considered significant.

\section{Results}

Figures 1 and 2 show that the groups were matched regarding age and $\operatorname{sex}(P=0.069$ and 0.714 , respectively). Table 1 shows that there were statistically significant differences among the three groups for hemoglobin $(P=0)$, uric acid $(P=0) \mathrm{HCO}_{3}$ $(P=0)$, eGFR $(P=0)$, urea $(P=0)$, and creatinine $(P=0)$. Post hoc analysis showed significant differences between the control and HD groups $(P=0)$ and the control and $\mathrm{CKD}$ groups on all laboratory tests $(P=0)$. The $\mathrm{HD}$ and $\mathrm{CKD}$ groups showed significant differencesfor $\mathrm{HCO}_{3}(P=0.002)$, eGFR $(P=0)$ urea $(P=0)$ and creatinine $(\mathrm{P}=0)$.

Table 2 shows that there were statistically significant differences among the three groups for VC $(P=0)$, FVC $(P=0), \quad \mathrm{FEV}_{1}(P=0), \quad \mathrm{FEV}_{1} / \mathrm{FVC}(P=0), \quad \mathrm{FEF} \quad \mathrm{FEF}_{25 \%}$ $(P=0)$, and $\mathrm{FEF}_{75 \%}(P=0)$. Post hoc analysis showed significant differences between the control and HD groups on all parameters $(P=0,0,0,0.030,0$, and 0 for $\mathrm{VC}, \mathrm{FVC}$, $\mathrm{FEV}_{1}, \mathrm{FEV}_{1} / \mathrm{FVC}, \mathrm{FEF}_{25 \%}$, and $\mathrm{FEF}_{75 \%}$, respectively) except expiratory time $(P=0.907)$ and significant differences between the control and $\mathrm{CKD}$ groups on all parameters $\left(P=0,0,0,0,0.010,0\right.$, and 0 for VC, FVC, $\mathrm{FEV}_{1}$, $\mathrm{FEV}_{1} / \mathrm{FVC}, \mathrm{FEF}_{25 \%}$, and $\mathrm{FEF}_{75 \%}$, respectively) except expiratory time $(P=0.606)$. The $\mathrm{HD}$ and $\mathrm{CKD}$ groups 


\section{Sex of the studied participants}



Female $\mathbf{m a l e}$

$P>0.05$

Figure I Sex of participants.

\section{Age of the studied participants}

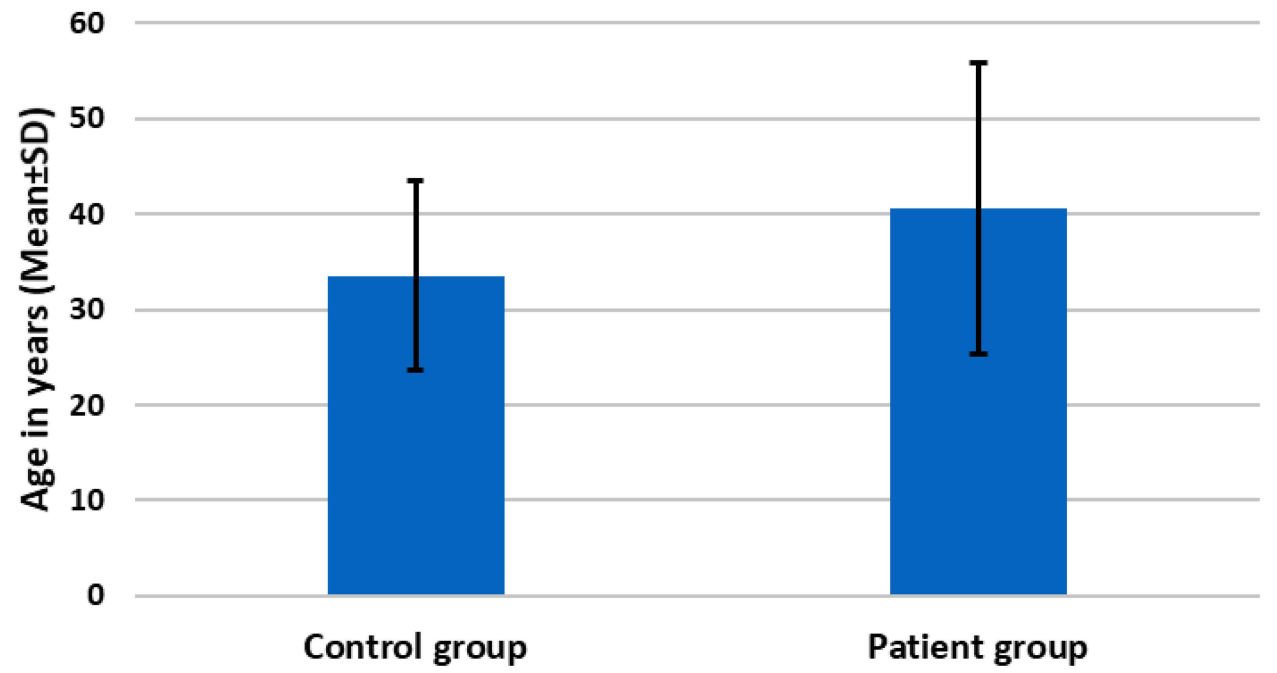

Figure 2 Age of participants.

Abbreviation: SD, Standard deviation.

showed nonsignificant differences on all parameters $(P=0.550,0.037,0.130,0.026,0.777,0.176$, and 0.498 for expiratory time, $\mathrm{VC}, \mathrm{FVC}, \mathrm{FEV}_{1}, \mathrm{FEV}_{1} / \mathrm{FVC}$, $\mathrm{FEF}_{25 \%}$, and $\mathrm{FEF}_{75 \%}$, respectively). Table 3 shows that there were statistically significant differences among the three groups for $f_{0}, S \%, J \%$, and HNR $(P=0.013,00$ and 0 , respectively). Post hoc analysis showed significant differences between the control and HD groups on all parameters $(P=0.018,00$ and 0 respectively) and the control and CKD groups on all parameters $(P=0.032,00.017$, and 0 respectively). The HD and CKD groups showed nonsignificant differenceson all parameters $(P=0.812$, $0.176,0.641$, and 0.050 , respectively).

Table 4 shows that there was a statistically significant positive correlation between duration of $\mathrm{HD}$ and HNR $(P=0$ and statistically significant negative correlation 
Table I Comparisons Among Control $(n=20), \mathrm{HD}(n=20)$, and CKD $(n=20)$ groups on Hemoglobin, Uric Acid, HCO 3 , eGFR, UreaReduction Ratio, Urea, and Creatinine

\begin{tabular}{|c|c|c|c|c|c|c|c|c|c|}
\hline & & \multirow{2}{*}{$\begin{array}{c}\text { Control } \\
n=20\end{array}$} & \multirow{2}{*}{$\begin{array}{l}\text { HD } \\
n=20\end{array}$} & \multirow{2}{*}{$\begin{array}{l}C K D \\
n=20\end{array}$} & \multirow[t]{2}{*}{$\mu^{*}$} & \multirow[t]{2}{*}{$P$} & \multicolumn{3}{|c|}{ Post hoc Analysis } \\
\hline & & & & & & & $P_{1}$ & $P_{2}$ & $P_{3}$ \\
\hline \multirow[t]{2}{*}{ Hemoglobin } & Mean \pm SD & $11.93 \pm 0.73$ & $9.78 \pm 1.35$ & $10.60 \pm 1.60$ & \multirow[t]{2}{*}{13.719} & \multirow[t]{2}{*}{0} & \multirow[t]{2}{*}{0} & \multirow[t]{2}{*}{0.002} & \multirow[t]{2}{*}{0.098} \\
\hline & Range & $10.5-13$ & $8-12$ & $8-13$ & & & & & \\
\hline \multirow[t]{2}{*}{ Uric acid } & Mean \pm SD & $3.77 \pm 0.60$ & $6.80 \pm 0.77$ & $7.10 \pm 0.79$ & \multirow[t]{2}{*}{129.715} & \multirow[t]{2}{*}{0} & \multirow[t]{2}{*}{0} & \multirow[t]{2}{*}{0} & \multirow[t]{2}{*}{0.230} \\
\hline & Range & $3-5$ & $6-8$ & $6-8$ & & & & & \\
\hline \multirow[t]{2}{*}{$\mathrm{HCO}_{3}$} & Mean \pm SD & $23.20 \pm 1.32$ & $18.60 \pm 1.31$ & $16.95 \pm 1.85$ & \multirow[t]{2}{*}{91.329} & \multirow[t]{2}{*}{0} & \multirow[t]{2}{*}{0} & \multirow[t]{2}{*}{0} & \multirow[t]{2}{*}{0.002} \\
\hline & Range & $21-26$ & $16-20$ & $14-20$ & & & & & \\
\hline \multirow[t]{2}{*}{ eGFR } & Mean \pm SD & $|16.25 \pm| 8.8 \mid$ & $8.10 \pm 2.95$ & $31.60 \pm 5.51$ & \multirow[t]{2}{*}{494.045} & \multirow[t]{2}{*}{0} & \multirow[t]{2}{*}{0} & \multirow[t]{2}{*}{0} & \multirow[t]{2}{*}{0} \\
\hline & Range & $79-142$ & $5-12$ & $23-40$ & & & & & \\
\hline \multirow{2}{*}{$\begin{array}{l}\text { Urea-reduction } \\
\text { ratio }\end{array}$} & Mean \pm SD & - & $64.60 \pm 6.84$ & - & \multirow[t]{2}{*}{ NA } & \multirow[t]{2}{*}{ NA } & & & \\
\hline & Range & - & $55-76$ & - & & & & & \\
\hline \multirow[t]{2}{*}{ Urea } & Mean \pm SD & $32.45 \pm 6.37$ & $177.30 \pm 20.09$ & $110.30 \pm 13.80$ & \multirow[t]{2}{*}{496.621} & \multirow[t]{2}{*}{0} & 0 & 0 & 0 \\
\hline & Range & $23-41$ & $140-200$ & $96-135$ & & & & & \\
\hline Creatinine & Mean \pm SD & $0.88 \pm 0.18$ & $8.80 \pm 0.89$ & $3.42 \pm 1.38$ & 358.480 & 0 & 0 & 0 & 0 \\
\hline & Range & $0.5-1.2$ & $8-11$ & $1.3-6$ & & & & & \\
\hline
\end{tabular}

Notes: *One-way ANOVA; $P_{1}$, control vs HD; $P_{2}$, control vs CKD; $P_{3}$, HD vs CKD.

Abbreviation: eGFR, estimated glomerular filtration rate.

Table 2 Comparisons Among Control $(n=20), H D(n=20)$, and CKD $(n=20)$ Groups on Respiratory Function Tests

\begin{tabular}{|c|c|c|c|c|c|c|c|c|c|}
\hline & & \multirow{2}{*}{$\begin{array}{c}\text { Control } \\
\mathrm{n}=\mathbf{2 0}\end{array}$} & \multirow{2}{*}{$\begin{array}{c}\text { HD } \\
n=20\end{array}$} & \multirow{2}{*}{$\begin{array}{l}\text { CKD } \\
n=20\end{array}$} & \multirow[t]{2}{*}{$\mu^{*}$} & \multirow[t]{2}{*}{$P$} & \multicolumn{3}{|c|}{ Post hoc Analysis } \\
\hline & & & & & & & $P_{1}$ & $\mathbf{P}_{2}$ & $P_{3}$ \\
\hline \multirow{2}{*}{$\begin{array}{l}\text { Expiratory time } \\
\text { (seconds) }\end{array}$} & Mean \pm SD & $3.21 \pm 1.01$ & $3.24 \pm 0.49$ & $3.35 \pm 0.65$ & \multirow[t]{2}{*}{0.194} & \multirow[t]{2}{*}{0.825} & \multirow[t]{2}{*}{0.907} & \multirow[t]{2}{*}{0.606} & \multirow[t]{2}{*}{0.550} \\
\hline & Range & $1.5-4.5$ & $2.4-4$ & $3-5$ & & & & & \\
\hline \multirow[t]{2}{*}{ VC } & Mean \pm SD & $106.50 \pm 11.98$ & $57.70 \pm 14.66$ & $67.30 \pm 13.39$ & \multirow[t]{2}{*}{74.559} & \multirow[t]{2}{*}{0} & \multirow[t]{2}{*}{0} & \multirow[t]{2}{*}{0} & \multirow[t]{2}{*}{0.037} \\
\hline & Range & $93-125$ & $47-85$ & $55-87$ & & & & & \\
\hline \multirow[t]{2}{*}{ FVC } & Mean \pm SD & $94.30 \pm 7.89$ & $55.90 \pm 14.48$ & $62.40 \pm 11.93$ & \multirow[t]{2}{*}{61.165} & \multirow[t]{2}{*}{0} & \multirow[t]{2}{*}{0} & \multirow[t]{2}{*}{0} & \multirow[t]{2}{*}{0.130} \\
\hline & Range & $83-112$ & $45-83$ & $45-85$ & & & & & \\
\hline \multirow[t]{2}{*}{$\mathrm{FEV}_{1}$} & Mean \pm SD & $97.55 \pm 8.38$ & $60.40 \pm 15.49$ & $70.60 \pm 12.16$ & \multirow[t]{2}{*}{48.283} & \multirow[t]{2}{*}{0} & \multirow[t]{2}{*}{0} & \multirow[t]{2}{*}{0} & \multirow[t]{2}{*}{0.026} \\
\hline & Range & $83-112$ & $49-90$ & $50-84$ & & & & & \\
\hline \multirow[t]{2}{*}{$\mathrm{FEV}_{1} / \mathrm{FVC}$} & Mean \pm SD & $1.04 \pm 0.12$ & $1.12 \pm 0.31$ & $1.20 \pm 0.10$ & \multirow[t]{2}{*}{1.296} & \multirow[t]{2}{*}{0} & \multirow[t]{2}{*}{0.030} & \multirow[t]{2}{*}{0.010} & \multirow[t]{2}{*}{0.777} \\
\hline & Range & $0.77-1.32$ & $0.69-1.96$ & $0.99-1.28$ & & & & & \\
\hline $\mathrm{FEF}_{25 \%}$ & Mean \pm SD & $7.32 \pm 0.98$ & $63.40 \pm 1.67$ & $64.50 \pm 3.15$ & $4,690.336$ & 0 & 0 & 0 & 0.176 \\
\hline & Range & $5.82-8.56$ & $60-65$ & $60-69$ & & & & & \\
\hline $\mathrm{FEF}_{75 \%}$ & Mean \pm SD & $2.79 \pm 0.57$ & $63.40 \pm 1.39$ & $63.70 \pm 1.38$ & $|7,73| .312$ & 0 & 0 & 0 & 0.498 \\
\hline & Range & $1.62-3.42$ & $62-65$ & $62-65$ & & & & & \\
\hline
\end{tabular}

Notes: *One-way ANOVA; $P_{1}$, control vs HD; $P_{2}$, control vs CKD; $P_{3}$, HD vs CKD.

Abbreviations: $\mathrm{FEV}_{1}$, forced expiratory volume in I second; FVC, forced vital capacity; FEF, forced expiratory flow. 
Table 3 Comparisons Among Control $(n=20), H D(n=20)$, and CKD $(n=20)$ Groups on Acoustic Parameters

\begin{tabular}{|c|c|c|c|c|c|c|c|c|c|}
\hline & & \multirow{2}{*}{$\begin{array}{c}\text { Control } \\
n=20\end{array}$} & \multirow{2}{*}{$\begin{array}{c}\text { HD } \\
n=20\end{array}$} & \multirow{2}{*}{$\begin{array}{l}\text { CKD } \\
n=20\end{array}$} & \multirow[t]{2}{*}{$\mu^{*}$} & \multirow[t]{2}{*}{$\boldsymbol{P}$} & \multicolumn{3}{|c|}{ Post hoc snalysis } \\
\hline & & & & & & & $P_{\mathbf{I}}$ & $P_{2}$ & $P_{3}$ \\
\hline Fundamental frequency & $\begin{array}{l}\text { Mean } \pm \text { SD } \\
\text { Range }\end{array}$ & $\begin{array}{c}144.15 \pm 47.14 \\
98-213\end{array}$ & $\begin{array}{c}177.02 \pm 35.77 \\
135.2-225.7\end{array}$ & $\begin{array}{c}\mid 74.23 \pm 37.74 \\
|27.9-2| 6.8\end{array}$ & 4.046 & 0.013 & 0.018 & 0.032 & 0.812 \\
\hline Shimmer percentage & $\begin{array}{l}\text { Mean } \pm \text { SD } \\
\text { Range }\end{array}$ & $\begin{array}{c}0.63 \pm 0.13 \\
0.5-0.99\end{array}$ & $\begin{array}{l}2.40 \pm 0.59 \\
1.84-3.75\end{array}$ & $\begin{array}{c}2.67 \pm 0.64 \\
1.95-3.4\end{array}$ & 93.492 & 0 & 0 & 0 & 0.176 \\
\hline Jitter percentage & $\begin{array}{l}\text { Mean } \pm \text { SD } \\
\text { Range }\end{array}$ & $\begin{array}{c}2.00 \pm 0.61 \\
0.55-2.8\end{array}$ & $\begin{array}{l}1.10 \pm 1.12 \\
0.43-3.98\end{array}$ & $\begin{array}{l}|.43 \pm 0.8| \\
0.39-2.77\end{array}$ & 3.732 & 0 & 0 & 0.017 & $0.64 I$ \\
\hline Harmonic:noise ratio & $\begin{array}{l}\text { Mean } \pm \text { SD } \\
\text { Range }\end{array}$ & $\begin{array}{c}7.80 \pm 0.23 \\
7.4-8.2\end{array}$ & $\begin{array}{c}6.29 \pm 1.22 \\
3.7-7.3\end{array}$ & $\begin{array}{c}6.87 \pm 0.40 \\
6.2-7.4\end{array}$ & 20.544 & 0 & 0 & 0 & 0.050 \\
\hline
\end{tabular}

Notes: *One-way ANOVA; $P_{1}$, control vs HD; $P_{2}$, control vs CKD; $P_{3}, H D$ vs CKD. Abbreviation: CKD, chronic kidney disease.

Table 4 Correlations between duration of HD and demographic data, laboratory tests, respiratory function tests, and acoustic parameters

\begin{tabular}{|c|c|c|}
\hline & \multicolumn{2}{|c|}{ Duration of HD } \\
\hline & $R$ & $P$ \\
\hline Age & -0.415 & 0.069 \\
\hline $\mathrm{Hb}$ & -0.178 & 0.479 \\
\hline Uric acid & 0.362 & 0.117 \\
\hline $\mathrm{HCO}_{3}$ & $-0.602 *$ & 0.005 \\
\hline eGFR & -0.362 & 0.117 \\
\hline Urea-reduction ratio & 0.266 & 0.257 \\
\hline Urea & -0.310 & 0.184 \\
\hline Creatinine & -0.311 & 0.182 \\
\hline Expiratory time (seconds) & -0.275 & 0.241 \\
\hline VC & $-0.579 *$ & 0.007 \\
\hline FVC & $-0.576 *$ & 0.008 \\
\hline $\mathrm{FEV}_{\mathrm{I}}$ & $-0.51 \mathrm{I} *$ & 0.021 \\
\hline $\mathrm{FEV}_{1} / \mathrm{FVC}$ & -0.019 & 0.936 \\
\hline $\mathrm{FEF}_{25 \%}$ & -0.334 & 0.151 \\
\hline $\mathrm{FEF}_{75 \%}$ & -0.123 & 0.605 \\
\hline Fundamental frequency & 0.066 & 0.783 \\
\hline Shimmer percentage & $-0.78 I^{*}$ & 0 \\
\hline Jitter percentage & $-0.823 *$ & 0 \\
\hline Harmonic:noise ratio & $0.850 *$ & 0 \\
\hline
\end{tabular}

Note: $* P<0.05$.

Abbreviations: $\mathrm{Hb}$, hemoglobin; eGFR, estimated glomerular filtration rate; $F E V_{1}$, forced expiratory volume in I second; FVC, forced vital capacity; FEF, forced expiratory flow.

between duration of $\mathrm{HD}$ and $S \%(\mathrm{P}=0)$ and duration of $\mathrm{HD}$ and $J \%(P=0)$.

Table 5 shows that in the HD group, there were statistically significant positive correlations between creatinine and $f_{0}, S h \%$, and $J \%$ percent $(P=0.002,0.014$, and 0.004 respectively) and a statistically significant negative correlation between creatinine and $\operatorname{HNR}(P=0.044)$. There was statistically significant positive correlations between expiratory time, VC, FVC, and $\mathrm{FEV}_{1}$ and $J \%(P=0.024$, $0.001,0.001$, and 0 respectively) and between $\mathrm{FEV}_{1}$ and $S$ $\%$ and $\mathrm{FEF}_{25 \%}$ and $S \%$ ( $P=0.002$ and 0.009 , respectively). There was statistically significant negative correlations between VC, FVC, $\mathrm{FEV}_{1}$, and $\mathrm{FEF}_{25 \%}$ and $\mathrm{HNR}$ ( $P=0.037,0.040,0$, and 0.022 , respectively).

Table 6 shows that in the CKD group, there were statistically significant positive correlations between hemoglobin, eGFR, FVC and FEV1 and HNR $(P=0.002$, $0,0.019$, and 0.022 respectively), and statistically significant negative correlations between both urea and creatinine and $\mathrm{HNR}$ ratio ( $P=0$ and 0.001 , respectively). It also reveals a statistically significant negative correlation between $\mathrm{HCO}_{3}$ and $f_{0}(P=0.020)$, and between expiratory time and $J \%(P=0.037)$. In addition to statistically significant negative correlation between $\mathrm{FEF}_{25 \%}$ and $S \%$ and between $\mathrm{FEF}_{75 \%}$ and $J \% \quad(\mathrm{P}=0.029 \quad$ and 0.024 respectively).

Table 7 shows that the most relevant significant factors predicting $f_{0}$ were $\mathrm{Hb}$, uric acid, eGFR, urea-reduction ratio, urea, expiratory time, and $\mathrm{FVC}$ and the most relevant significant factors predicting $J \%$ were $\mathrm{Hb}$, uric acid, $\mathrm{HCO}_{3}$, urea-reduction ratio, creatinine, expiratory time, $\mathrm{VC}$, and $\mathrm{FEF}_{25 \%}$. It also shows that the most relevant significant factors predicting $S \%$ were uric acid, eGFR, urea-reduction ratio, urea, expiratory time, $\mathrm{FVC}, \mathrm{FEV}_{1} /$ $\mathrm{FEV}_{5}$, and $\mathrm{FEF}_{75 \%}$ and the most relevant significant factors predicting $\mathrm{HNR}$ were $\mathrm{Hb}, \mathrm{HCO}_{3}$, eGFR, creatinine, $\mathrm{FVC}, \mathrm{FEV}_{1} / \mathrm{FEV}_{5}, \mathrm{FEF}_{25 \%}$, andFEF $25 \%$. Regression analysis revealed that hemoglobin, uric acid, and expiratory time were the most significant predictors for impaired acoustic characteristics. 
Table 5 Correlations Between Laboratory Tests and Respiratory Function Tests Against Acoustic Parameters in the HD Group

\begin{tabular}{|c|c|c|c|c|c|c|c|c|}
\hline & \multicolumn{2}{|c|}{ Fundamental Frequency } & \multicolumn{2}{|c|}{ Shimmer Percentage } & \multicolumn{2}{|c|}{ Jitter Percentage } & \multicolumn{2}{|c|}{ Harmonic:Noise Ratio } \\
\hline & $\boldsymbol{r}$ & $\mathbf{P}$ & $\boldsymbol{r}$ & $P$ & $\boldsymbol{r}$ & $\boldsymbol{P}$ & $r$ & $\mathbf{P}$ \\
\hline $\mathrm{Hb}$ & 0.369 & 0.132 & -0.073 & 0.773 & 0.153 & 0.544 & -0.044 & 0.863 \\
\hline Uric acid & -0.242 & 0.303 & -0.156 & 0.512 & -0.107 & 0.654 & 0.278 & 0.235 \\
\hline $\mathrm{HCO}_{3}$ & 0.191 & 0.421 & 0.365 & 0.114 & 0.394 & 0.085 & -0.403 & 0.078 \\
\hline eGFR & -0.129 & 0.588 & 0.323 & 0.165 & 0.217 & 0.357 & -0.297 & 0.203 \\
\hline Urea-reduction ratio & 0.202 & 0.394 & -0.127 & 0.593 & 0.078 & 0.745 & -0.032 & 0.893 \\
\hline Urea & -0.255 & 0.278 & 0.347 & 0.134 & 0.383 & 0.095 & -0.250 & 0.288 \\
\hline Creatinine & $0.638 *$ & 0.002 & $0.539 *$ & 0.014 & $0.613^{*}$ & 0.004 & $-0.455^{*}$ & 0.044 \\
\hline Expiratory time (seconds) & 0.423 & 0.063 & 0.181 & 0.444 & $0.502 *$ & 0.024 & -0.217 & 0.357 \\
\hline VC & 0.369 & 0.110 & 0.416 & 0.068 & $0.694^{*}$ & 0.001 & $-0.469 *$ & 0.037 \\
\hline FVC & 0.364 & 0.114 & 0.409 & 0.073 & $0.693 *$ & 0.001 & $-0.462 *$ & 0.040 \\
\hline $\mathrm{FEV}_{1}$ & 0.170 & 0.475 & $0.658 *$ & 0.002 & $0.836 *$ & 0 & $-0.827 *$ & 0 \\
\hline $\mathrm{FEV}_{1} / \mathrm{FVC}$ & -0.283 & 0.227 & 0.216 & 0.362 & 0.198 & 0.403 & -0.383 & 0.096 \\
\hline $\mathrm{FEF}_{25 \%}$ & -0.121 & $0.6 \mathrm{II}$ & $0.567 *$ & 0.009 & 0.438 & 0.053 & $-0.510 *$ & 0.022 \\
\hline $\mathrm{FEF}_{75 \%}$ & 0.015 & 0.951 & 0.435 & 0.055 & 0.384 & 0.095 & -0.443 & 0.050 \\
\hline
\end{tabular}

Note: $* P<0.05$.

Abbreviations: $\mathrm{Hb}$, hemoglobin; CKD, chronic kidney disease; eGFR, estimated glomerular filtration rate; FEV capacity; FEF, forced expiratory flow.

Table 6 Correlations between Laboratory Tests and Respiratory Function Tests Against Acoustic Parameters in the CKD Group

\begin{tabular}{|c|c|c|c|c|c|c|c|c|}
\hline & \multicolumn{2}{|c|}{ Fundamental Frequency } & \multicolumn{2}{|c|}{ Shimmer Percentage } & \multicolumn{2}{|c|}{ Jitter Percentage } & \multicolumn{2}{|c|}{ Harmonic:Noise Ratio } \\
\hline & $\boldsymbol{r}$ & $P$ & $\boldsymbol{r}$ & $\boldsymbol{P}$ & $r$ & $P$ & $r$ & $\boldsymbol{P}$ \\
\hline $\mathrm{Hb}$ & 0.021 & 0.930 & -0.144 & 0.545 & -0.084 & 0.725 & $0.660 *$ & 0.002 \\
\hline Uric acid & 0.051 & 0.830 & 0.319 & 0.171 & 0.061 & 0.798 & -0.072 & 0.763 \\
\hline $\mathrm{HCO}_{3}$ & $-0.514 *$ & 0.020 & -0.121 & 0.612 & -0.355 & 0.124 & -0.248 & 0.292 \\
\hline eGFR & 0.165 & 0.486 & -0.357 & 0.122 & -0.197 & 0.404 & $0.73 I^{*}$ & 0 \\
\hline Urea & -0.054 & 0.820 & $-0.14 \mid$ & 0.553 & 0.238 & 0.311 & $-0.716^{*}$ & 0 \\
\hline Creatinine & 0.079 & 0.742 & -0.372 & 0.106 & -0.139 & 0.560 & $-0.666 *$ & 0.001 \\
\hline Expiratory time (seconds) & -0.362 & 0.117 & -0.122 & 0.609 & $-0.468^{*}$ & 0.037 & 0.162 & 0.495 \\
\hline VC & -0.272 & 0.245 & 0.418 & 0.067 & -0.131 & 0.582 & 0.376 & 0.103 \\
\hline FVC & -0.323 & 0.165 & 0.152 & 0.523 & -0.159 & 0.503 & $0.521^{*}$ & 0.019 \\
\hline $\mathrm{FEV}_{\text {, }}$ & -0.277 & 0.237 & 0.423 & 0.063 & 0.046 & 0.846 & $0.510 *$ & 0.022 \\
\hline $\mathrm{FEV}_{1} / \mathrm{FVC}$ & 0.051 & 0.832 & 0.391 & 0.088 & 0.315 & 0.176 & $-0.07 \mid$ & 0.766 \\
\hline $\mathrm{FEF}_{25 \%}$ & -0.195 & 0.411 & -0.098 & 0.682 & 0.073 & 0.759 & 0.017 & 0.943 \\
\hline $\mathrm{FEF}_{75 \%}$ & 0.191 & 0.421 & $-0.488^{*}$ & 0.029 & $-0.503^{*}$ & 0.024 & 0.113 & 0.636 \\
\hline
\end{tabular}

Note: $* P<0.05$.

Abbreviations: $\mathrm{Hb}$, hemoglobin; eGFR, estimated glomerular filtration rate; FEV flow.

\section{Discussion}

Dysphonia has been reported among chronic kidney disease and hemodialyzed patients. However, its exact etiology has been little discussed. For optimal vibration to occur, the vocal folds need sufficient hydration, both systemic within the tissues of the larynx and superficial to its lumen. The kidneys play the major role in maintaining and balancing hydration within the human body, and their impairment will in turn affect voice production.
Sivasankar and Leydon assumed that vocal hygiene and phonatory competence improved with adequate hydration. ${ }^{9}$

All patients in the current study underwent laboratory investigations and respiratory function tests. Laboratory investigations were $\mathrm{Hb}$, uric acid, $\mathrm{HCO}_{3}$, eGFR, ureareduction ratio, urea, and creatinine. Urea-reduction ratio was used to assess the efficiency of HD before voice analysis. If the HD had been inefficient, the voice analysis 
Table 7 Linear Regression for Predictors of Changes of Acoustic Parameters

\begin{tabular}{|c|c|c|c|c|c|c|c|c|}
\hline & \multicolumn{2}{|l|}{$\begin{array}{l}\text { Fundamental } \\
\text { Frequency }\end{array}$} & \multicolumn{2}{|l|}{ Jitter Percentage } & \multicolumn{2}{|c|}{ Shimmer Percentage } & \multicolumn{2}{|c|}{ Harmonic:Noise Ratio } \\
\hline & $\begin{array}{l}\text { Unstandardized } \\
\beta \text { Coefficient }\end{array}$ & $P$ & $\begin{array}{l}\text { Unstandardized } \\
\beta \text { Coefficient }\end{array}$ & $P$ & $\begin{array}{l}\text { Unstandardized } \\
\beta \text { Coefficient }\end{array}$ & $P$ & $\begin{array}{l}\text { Unstandardized } \\
\beta \text { coefficient }\end{array}$ & $P$ \\
\hline $\mathrm{Hb}$ & -52.236 & $0 *$ & 0.488 & $0 *$ & & & 0.404 & $0 *$ \\
\hline Uric acid & -27.697 & $0 *$ & 0.888 & $0 *$ & 0.829 & $0 *$ & & \\
\hline $\mathrm{HCO} 3$ & & & 0.437 & $0 *$ & & & -0.162 & $0 *$ \\
\hline eGFR & 3.411 & $0^{*}$ & & & -0.200 & $0^{*}$ & -0.077 & $0 *$ \\
\hline Urea-reduction ratio & 4.597 & $0^{*}$ & 0.058 & $0 *$ & -0.105 & $0^{*}$ & & \\
\hline Urea & -2.702 & $0 *$ & & & -0.008 & $0^{*}$ & & \\
\hline Creatinine & & & 0.912 & $0 *$ & & & -0.347 & $0 *$ \\
\hline Expiratory time (seconds) & 43.650 & $0 *$ & -1.690 & $0 *$ & -0.273 & $0^{*}$ & & \\
\hline VC & & & 0.045 & $0 *$ & & & & \\
\hline FVC & 2.630 & $0 *$ & & & -0.040 & $0^{*}$ & -0.025 & $0^{*}$ \\
\hline $\mathrm{FEV}_{\mathrm{I}}$ & & & & & & & & \\
\hline $\mathrm{FEV}_{1} / \mathrm{FVC}$ & & & & & 0.285 & $0^{*}$ & -0.082 & $0 *$ \\
\hline $\mathrm{FEF}_{25 \%}$ & & & 0.114 & $0 *$ & & & -0.089 & $0.002 *$ \\
\hline $\mathrm{FEF}_{75 \%}$ & & & & & -0.414 & $0^{*}$ & 0.211 & $0.001 *$ \\
\hline Constant & -752.85 & $0 *$ & -26.559 & $0 *$ & 11.297 & $0^{*}$ & 9.915 & $0.015^{*}$ \\
\hline
\end{tabular}

Note: $* P<0.05$.

Abbreviations: $\mathrm{Hb}$, hemoglobin; eGFR, estimated glomerular filtration rate; FEV, forced expiratory volume in I second; FVC, forced vital capacity; FEF, forced expiratory flow.

would be postponed. eGFR was used to assess residual kidney function in HD patients because it is more convenient than isotope scans. The results of the current study showed significant increases in uric acid, urea, and creatinine and significant decreases in $\mathrm{Hb}, \mathrm{HCO}_{3}$, and eGFR in all patients in comparison to the normal group. The increases in urea and creatinine were responsible for increases in $f_{0}$ and $S \%$, in patients on $\mathrm{HD}$, and increase in urea and creatinine together with decreases in $\mathrm{Hb}$ and eGFR were responsible for the reduction in HNR in patients with CKD. Similarly, Sagiroglu and Doganer declared that increases uric acid, creatinine, and $\mathrm{Na}^{+}$are most probably responsible for changes in vocal parameters in patients with renal failure, particularly after HD. ${ }^{10}$

Cases et al reported that patients with reduced eGFR frequently suffer from anemia. ${ }^{11}$ They proposed that anemia may be responsible in part for the generalized muscle weakness and fatigue in those patients. In addition, $\mathrm{HCO}_{3}$ reflects the degree of regulation of extracellular fluid buffering. The lungs and kidneys cooperate to maintain this system. ${ }^{12}$ Reduction in $\mathrm{HCO}_{3}$ induces hyperventilation (respiratory alkalosis) and subsequently impairs respiratory function. ${ }^{13}$ Patients in the current study showed significant restrictive patterns in respiratory function (reduced $\mathrm{FVC}$, increased mean $\mathrm{FEV}_{1} / \mathrm{FVC}$, and increased mean
FEF), with nonsignificant difference between those with CKD and those on HD. Mukai et al postulated that 36\%$79 \%$ of patients with CKD may show restrictive respiratory patterns. ${ }^{14}$ Hassan performed acoustic and aerodynamic analysis of the voices of patients with CKD. ${ }^{15}$ She found that they exhibited higher $f_{0}$ and $S \%$ and lower HNR and maximal phonation time than normal. Maximal phonation time is affected by reductions in respiratory and/or glottal efficiency; however, it cannot differentiate between them. In the current study, $\mathrm{FEV}_{1}$ was found to be responsible for increased $J \%$ and $S h \%$ and decreased HNR in patients on HD. Reduced VC and FVC were responsible for increased $J \%$ in patients on HD. On the contrary, Kumar and Clark noted that increased $f_{0}$ in male patients may occur due to reduced testosterone levels as a result of CKD. ${ }^{16}$

Acoustic voice analysis is the least invasive and most convenient and objective valid measure of vocal parameters. Any change in the vocal fold mass and tension affects the acoustic characteristics of voice.

All patients in the current study showed significant changes in all acoustic parameters when compared to the control group (increase in $f_{0}, S \%, J \%$, and reduction in HNR: $P=0.013,0,0$, and 0, respectively). Differences between patients with $\mathrm{CKD}$ and patients on HD was 
nonsignificant on all acoustic parameters $(P=0.812,0.176$, 0.641 , and 0.050 , respectively). Similarly, Unver et al studied the effect of HD on voice quality and found that all patients had significant increases in $f_{0}$ and HNR after HD. ${ }^{17}$ They attributed these findings to ultrafiltration and changes in extracellular fluid volume. According to Raphael et al, changes in the vibratory patterns of vocal folds after HD could be attributed to changes in their length, mass, and tension. ${ }^{18}$ They proposed that the muscles function normally after HD. On the other hand, Nesić et al recorded no changes in $f_{0}$ after HD. ${ }^{19}$

The results of the current study show that the changes in voice observed in patients with $\mathrm{CKD}$ and patients on HD resulted from a combination of impaired hydration, acid-base imbalance, circulating toxins, and weakness of the muscles of respiration and phonation. They also reveal that HD has a detrimental effect on voice quality, both instantly after the session and in the long term, as this effect increases with the duration of HD.

\section{Conclusion}

The systemic effect of CKD and HD were found to impair the acoustic characteristics of voice in both groups. Regression analysis revealed that $\mathrm{Hb}$, uric acid, and expiratory time were the most significant predictors of impaired acoustic characteristics.

\section{Ethics Approval and Informed Consent}

This study was approved by the Research Ethics Committee of the Faculty of Medicine, Al Azhar University. All participants were informed about the study, and written informed consent was obtained. The study was conducted in accordance with the Declaration of Helsinki.

\section{Funding}

There was no funding provided.

\section{Disclosure}

The authors report no conflict of interest in this work.

\section{References}

1. Sivasankar M, Erickson E. Short-duration accelerated breathing challenges affect phonation. Laryngoscope. 2009;119(8):1658-1663. doi:10.1002/lary.20530

2. Adrogué HE, Adrogue HJ. Acid-base physiology. Respir Care. 2001;46(4):328-341.

3. Karacan Ö, Tutal E, Colak T, Sezer S, Eyüboğlu FÖ, Haberal M Pulmonary function in renal transplant recipients and end-stage renal disease patients undergoing maintenance dialysis. In Transplantation proceedings. Vol. 38. No. 2. Elsevier; 2006:396-400.

4. Pierson DJ. Respiratory considerations in the patient with renal failure. Respir Care. 2006;51(4):413-422.

5. Hamdan AL, Medawar W, Younes A, Bikhazi H, Fuleihan N. The effect of hemodialysis on voice: an acoustic analysis. $J$ Voice. 2005;19(2):290-295. doi:10.1016/j.jvoice.2004.01.011

6. Zumrutdal A. An overlooked complication of hemodialysis: Hoarseness. Hemodial Int. 2013;17(4):633-638. doi:10.1111/hdi.12028

7. Levey AS, Coresh J, Greene T, et al. Using standardized serum creatinine values in the modification of diet in renal disease study equation for estimating glomerular filtration rate. Ann Intern Med. 2006;145:247-254. doi:10.7326/0003-4819-145-4-200608150-00004

8. IBM Corp. IBM SPSS Statistics for Windows, Version 27.0. Armonk, NY: IBM Corp; 2020.

9. Sivasankar M, Leydon C. The role of hydration in vocal fold physiology. Curr Opin Otolaryngol Head Neck Surg. 2010;18(3):171. doi:10.1097/MOO.0b013e3283393784

10. Sagiroglu S, Doganer A. The effect of electrolyte balance on the voice in hemodialysis patients. Eur Arch Otorhinolaryngol. 2018;275 (11):2755-2761. doi:10.1007/s00405-018-5098-x

11. Cases A, Egocheaga MI, Tranche S, et al. Anemia of chronic kidney disease: protocol of study, management and referral to Nephrology. Nefrología (English Edition). 2018;38(1):8-12. doi:10.1016/j. nefroe.2018.01.007

12. Uchino S, Kellum JA, Bellomo R, et al. Acute renal failure in critically ill patients: a multinational, multicenter study. JAMA. 2005;294(7):813-818. doi:10.1001/jama.294.7.813

13. Li H, Li N. Pulmonary function tests and blood gas analysis. In: Li H, Li N, editors. Radiology of Influenza A (H1N1). Dordrecht: Springer; 2013:19-22. doi:10.1007/978-94-007-6162-9_7

14. Mukai H, Ming P, Lindholm B, et al. Lung dysfunction and mortality in patients with chronic kidney disease. Kidney Blood Press Res. 2018;43(2):522-535. doi:10.1159/000488699

15. Hassan ES. Effect of chronic renal failure on voice: an acoustic and aerodynamic analysis. Egypt J Otolaryngol. 2014;30(1):53-57. doi:10.4103/1012-5574.127207

16. Kumar P, Clark M. Clinical Medicine: A Textbook for Medical Students and Doctors. 4th ed. London: W.B. Saunders; 1999.

17. Unver S, Hardal U, Esertas K, Sezen A, Celikbilek F, Altundag A. Objective analysis of voice changes in a hemodialysis session and its correlation with ultrafiltration. Ren Fail. 2015;37(2):268-272. doi:10.3109/0886022X.2014.988108

18. Raphael LJ, Borden GJ, Harris KS. Speech Science Primer: Physiology, Acoustics, and Perception of Speech. 5th ed. Philadelphia: Lippincott Williams \& Wilkins; 2007:85-103.

19. Nesić M, Veljković S, Obrenović J, Cekić S, Velicković D, Radenković M. Voice frequencies in patients treated with chronic hemodialysis. Srp Arc Celok Lek. 1996;124:99-101. 


\section{Publish your work in this journal}

The International Journal of General Medicine is an international, peer-reviewed open-access journal that focuses on general and internal medicine, pathogenesis, epidemiology, diagnosis, monitoring and treatment protocols. The journal is characterized by the rapid reporting of reviews, original research and clinical studies across all disease areas. The manuscript management system is completely online and includes a very quick and fair peer-review system, which is all easy to use. Visit http://www.dovepress.com/ testimonials.php to read real quotes from published authors.

Submit your manuscript here: https://www.dovepress.com/international-journal-of-general-medicine-journal 